\title{
A Survey of Seyfert AGN: Nuclear Gas Disks and Direct Black Hole Mass Estimates
}

\author{
E. K. S. Hicks ${ }^{1}$, R. I. Davies ${ }^{1}$, M. A. Malkan ${ }^{2}$, \\ R. Genzel ${ }^{1,3}$, and L. J. Tacconi ${ }^{1}$ \\ ${ }^{1}$ Max Planck Institut für extraterrestrische Physik, Garching, Germany \\ Email: ehicks@mpe.mpg.de \\ ${ }^{2}$ Department of Physics and Astronomy, University of California, Los Angeles, USA \\ ${ }^{3}$ Department of Physics, University of California, Berkeley, USA
}

\begin{abstract}
In a survey of 18 nearby Seyfert nuclei, we find evidence for geometrically thick gas disks on scales of tens of parsecs. Mapping the interstellar medium traced by $\mathrm{H}_{2} \nu=1-$ 0S(1) emission using the infrared integral field spectrometers OSIRIS and SINFONI reveals general disk rotation with an additional significant component of random bulk motion implied by the high local velocity dispersion. The size scale of the typical nuclear gas disk is $\sim 30 \mathrm{pc}$ in radius with a comparable vertical height, and the distribution and kinematics suggest the gas is spatially mixed with the nuclear stellar population. Based on the estimated characteristic gas mass fraction of $10 \%$, the average gas mass within this region is $\sim 10^{7} M_{\odot}$. This suggests column densities of $N_{\mathrm{H}} \sim 5 \times 10^{23} \mathrm{~cm}^{-2}$, but the significantly lower densities implied by the stellar continuum extinction indicate that the gas distribution on these scales is dominated by dense clumps. We discuss the feasibility of constraining the masses of the central black holes via modeling of the gas disk kinematics, highlighting the importance of properly accounting for the gas velocity dispersion, and the use of these direct mass estimates to calibrate masses derived from the method of reverberation mapping.
\end{abstract}

Keywords. galaxies: active, galaxies: ISM, galaxies: kinematics and dynamics, galaxies: nuclei, galaxies: Seyfert, infrared: galaxies

\section{Introduction}

By tracing their gravitational potential via nuclear gas or stars, it has been shown that most, if not all, galaxies with a massive spheroidal component contain a massive dark central component at their centers, presumably a supermassive black hole (BH; e.g., Kormendy \& Richstone 1995). Furthermore, such studies have revealed that the black hole mass $\left(M_{\mathrm{BH}}\right)$ is correlated with properties of the host galaxy, such as bulge luminosity and $\sigma$ (the stellar velocity dispersion; e.g. Ferrarese \& Merritt 2000, Gebhardt et al. 2000, Gultekin et al. 2009). This implies that although a BH's gravitational influence reaches only the inner region of the host galaxy, its formation and evolution, assumed to occur in an active galactic nuclei (AGN) phase, are strongly coupled with that of the host galaxy. Study of the demographics of $M_{\mathrm{BH}}$ in AGN is therefore critical to understanding the role BHs play in galaxy evolution. Measurement of $M_{\mathrm{BH}}$ in AGN is also essential to understanding the AGN phenomenon, since $M_{\mathrm{BH}}$ is the fundamental parameter in all AGN theories and models.

It is challenging to directly measure $M_{\mathrm{BH}}$ in AGN because of the bright non-stellar emission from the nucleus, which dilutes the gas and stellar spectral signatures typically relied upon for such measurements. Alternatively, the method of reverberation mapping (Peterson et al. 2004) can measure $M_{\mathrm{BH}}$ in AGN, but because of the unknown broad line region (BLR) geometry and kinematics, questions remain about the potential systematic 
uncertainties of these estimates. In addition, secondary methods, such as the use of restframe ultraviolet luminosity and emission-line width, which has been shown to correlate with reverberation-mapped $M_{\mathrm{BH}}$ (e.g. Vestergaard 2002, McGill et al. 2008), are powerful in that they can measure $M_{\mathrm{BH}}$ with ease in large samples of AGN out to large redshifts. However, they are calibrated against the reverberation mapping results, and are thus even more uncertain. Validation and calibration of the reverberation mapping method would therefore provide a foundation for an " $M_{\mathrm{BH}}$ scale."

We have therefore begun a campaign to directly measure $M_{\mathrm{BH}}$ in AGN, with an emphasis on galaxies with $\mathrm{BH}$ mass estimates obtained via reverberation mapping. Such a goal is now possible by taking advantage of the relatively low nuclear AGN emission in the near-infrared, the high spatial resolutions achievable at these wavelengths with adaptive optics (AO), and the availability of integral field units (IFUs) with which the full two-dimensional (2-D) nuclear gas and stellar kinematics can be measured. In this paper we focus on the viability of obtaining reliable $M_{\mathrm{BH}}$ estimates from the nuclear gas kinematics; however, we are also currently working on obtaining $M_{\mathrm{BH}}$ estimates via modeling of the stellar kinematics. The relative ease with which one can measure the nuclear gas emission and model the kinematics compared to that of the nuclear stars makes it a relatively efficient approach, but it must first be demonstrated that the nuclear gas is a reliable tracer of the gravitational potential and is not significantly influenced by non-gravitational forces. As a first step toward assessing the potential of using the gas kinematics to obtain $M_{\mathrm{BH}}$ estimates, we characterize in detail the nuclear molecular gas in a sample of Seyfert galaxies, including a comparison of the kinematics to that of the nuclear stars. In the last section, we then discuss the feasibility of reliably modeling the gas kinematics to constrain $M_{\mathrm{BH}}$ in this critical sample of galaxies.

\section{Observations of the Central $\sim 100$ pc in a Sample of Seyferts}

Using near-infrared IFUs with OSIRIS on the Keck Telescope and SINFONI on the VLT, we probe both neutral and ionized gas, as well as the stellar population, throughout the central $\sim 100 \mathrm{pc}$ in a sample of 18 Seyfert galaxies. By using AO, spatial resolutions down to $4 \mathrm{pc}$ were achieved, with an average resolution of $42 \pm 37 \mathrm{pc}$ for the sample. With $R \sim 3000-4300 K$-band spectra we trace the nuclear gas with $\mathrm{H}_{2} \nu=1-0 \mathrm{~S}(1)$ and several ionized emission lines such as $\operatorname{Br} \gamma$, and the stellar population with the $2.3 \mu \mathrm{m} \mathrm{CO}$ absorption bandheads. Additional details of the observations and a full description of the data reduction steps are provided by Hicks et al. (2009). Table 1 provides a summary of the sample galaxies.

The sample of galaxies was selected based on (1) the availability of a black hole mass estimate from reverberation mapping, (2) the brightness of the AGN such that the nucleus could be used either as the guide star with natural guide star AO or as a tip-tilt guide with laser guide star AO, and (3) the distance of the galaxy such that at least 2.5 times the sphere of influence of the $\mathrm{BH}$ (based on reverberation-mapping mass estimates) can be resolved and the $\mathrm{H}_{2}$ 1-0 S(1) and CO bandheads are observable within the $K$-band. The first criterion restricts the sample to Seyfert 1 galaxies, however an additional three galaxies which have not been reverberation mapped - two Seyfert 2s, and a Seyfert 1/LINER - were also observed with SINFONI and are included in the sample.

\section{General Properties of $\mathbf{H}_{2}$ 1-0 S(1) in Seyfert Galaxies}

An analysis of the molecular hydrogen in a subsample of these Seyfert galaxies was presented by Hicks et al. (2009). We extend this analysis to the full sample of 18 galaxies 
Table 1. Summary of Seyfert Galaxy Sample.

\begin{tabular}{lcccccc}
\hline Galaxy & $\begin{array}{c}\text { PSF FWHM } \\
(\mathrm{pc})\end{array}$ & $\begin{array}{c}R^{1} \\
(\mathrm{pc})\end{array}$ & $\begin{array}{c}v_{\mathrm{rot}} \\
\left(\mathrm{km} \mathrm{s}^{-1}\right)\end{array}$ & $\begin{array}{c}\sigma \\
\left(\mathrm{km} \mathrm{s}^{-1}\right)\end{array}$ & $\begin{array}{c}M_{\mathrm{dyn}}^{2} \\
\left(10^{7} M_{\odot}\right)\end{array}$ & $\begin{array}{c}M_{\mathrm{dyn}}{ }^{3} \\
\left(10^{7} M_{\odot}\right)\end{array}$ \\
\hline NGC 3227 & 6 & 8 & 23 & 84 & 0.1 & 4.2 \\
NGC 3516* & 32 & 34 & 93 & - & 6.8 & - \\
NGC 4051 & 6 & 7 & 13 & 67 & 0.3 & 2.2 \\
NGC 4151 & 8 & 8 & 31 & 21 & 0.2 & 4.4 \\
NGC 4593 & 12 & 13 & 141 & 67 & 6.2 & 10.3 \\
NGC 5548* & 59 & 61 & 32 & - & 1.5 & - \\
NGC 6814 & 18 & 18 & 17 & 47 & 0.1 & 2.7 \\
NGC 7469 & 35 & 35 & 52 & 63 & 2.2 & 11.6 \\
Mrk 79 & 75 & 83 & 46 & 80 & 4.1 & 41.7 \\
Mrk 110 & 75 & 77 & 35 & 39 & 2.2 & 11.1 \\
Mrk 590 & 96 & 100 & 32 & - & 2.4 & - \\
Mrk 817 & 123 & 145 & 44 & 71 & 7.9 & 69 \\
Akn 120* & 90 & 95 & 278 & - & 170 & - \\
IC 4329A & 59 & 222 & 212 & 79 & 233 & 329 \\
NGC 3783 & 36 & 37 & 42 & 35 & 1.4 & 4.3 \\
\hline NGC 1097 & 22 & 24 & 51 & 52 & 1.5 & 6.0 \\
Circinus & 4 & 4 & 29 & 54 & 0.1 & 0.9 \\
NGC 1068 & 6 & 6 & 23 & 41 & 0.1 & 0.8 \\
\hline
\end{tabular}

Notes: All galaxies are Seyfert 1s with reverberation mapping black hole masses, except NGC 1097, which is a Seyfert 1/LINER, and NGC 1068 and Circinus, which are both Seyfert 2s. $v_{\text {rot }}$ are inclination-corrected values, and kinematics for those marked with * are from stars because $\mathrm{H}_{2}$ emission was not well detected.

${ }^{1}$ Radius at which $\Delta v$ and $\sigma$ are measured, which are used to derive the $M_{\mathrm{dyn}}$ estimates.

${ }^{2} M_{\mathrm{dyn}}$ estimates based only on the velocity gradient, $M_{d y n}=v_{\text {rot }}^{2} R / G$.

${ }^{3} M_{\mathrm{dyn}}$ estimates based on both $\Delta v$ and $\sigma$ as discussed in the text.

by now including 9 new Seyfert galaxies, 4 of which have well-detected $\mathrm{H}_{2}$ emission (see Figure 1 for 2-D maps of two of these galaxies). As discussed in detail below, with this expanded sample, we reach the same general conclusions regarding the state of the molecular gas on scales of tens of parsecs in Seyfert galaxies: when detected, the nuclear gas is in a geometrically and (often) optically thick disk that is spatially mixed with the nuclear stellar population.

The majority of galaxies observed have a relatively symmetric 2-D $\mathrm{H}_{2}$ flux distribution (the only significant exceptions being NGC 3227 and NGC 1068). The centrally concentrated nuclear molecular gas has an observed half-width-at-half-maximum (HWHM) less than $35 \mathrm{pc}$ in all cases where the nuclear emission is well resolved (Figure 2), and in those cases where the emission is poorly resolved the HWHM is $35 \mathrm{pc}$ or less after accounting for the point-spread-function (PSF). Sersic profile fits to the azimuthally averaged flux distributions indicate disk-like morphologies. Although this symmetry of the flux distribution may be somewhat surprising given the sensitivity of $\mathrm{H}_{2}$ emission to, for example, local star formation, this is consistent with our general conclusion that the ISM traced by $\mathrm{H}_{2}$ 1-0 $\mathrm{S}(1)$ emission is in a nuclear disk dominated by the gravitational potential.

The regularity of the velocity fields suggest the kinematics of the molecular gas is dominated by disk rotation, and that even in those cases where an additional kinematic component is identified, there is still an underlying rotating gas disk. Of note is the significant velocity dispersion of the gas, which implies substantial vertically thick disks. On average for the sample, $v_{\text {rot }} / \sigma<1$ out to, and in many cases well beyond, the HWHM radius (Figure 2). We estimate the disk heights based on the observed velocity dispersion assuming two scenarios: the nuclear gas and stars are self gravitating $\left(z_{o}=\sigma^{2} / 2 \pi G \Sigma\right.$, where $z_{o}$ is disk height, $\sigma$ the velocity dispersion, $G$ the gravitational constant, and $\Sigma$ the surface density of the disk) or vertical hydrostatic equilibrium $\left(v_{\text {rot }} / \sigma \sim R / z_{o}\right)$. Both estimates imply disk heights that are comparable to the disk radius. Hicks et al. (2009) explored several mechanisms as a potential source of support for these thick disks and 

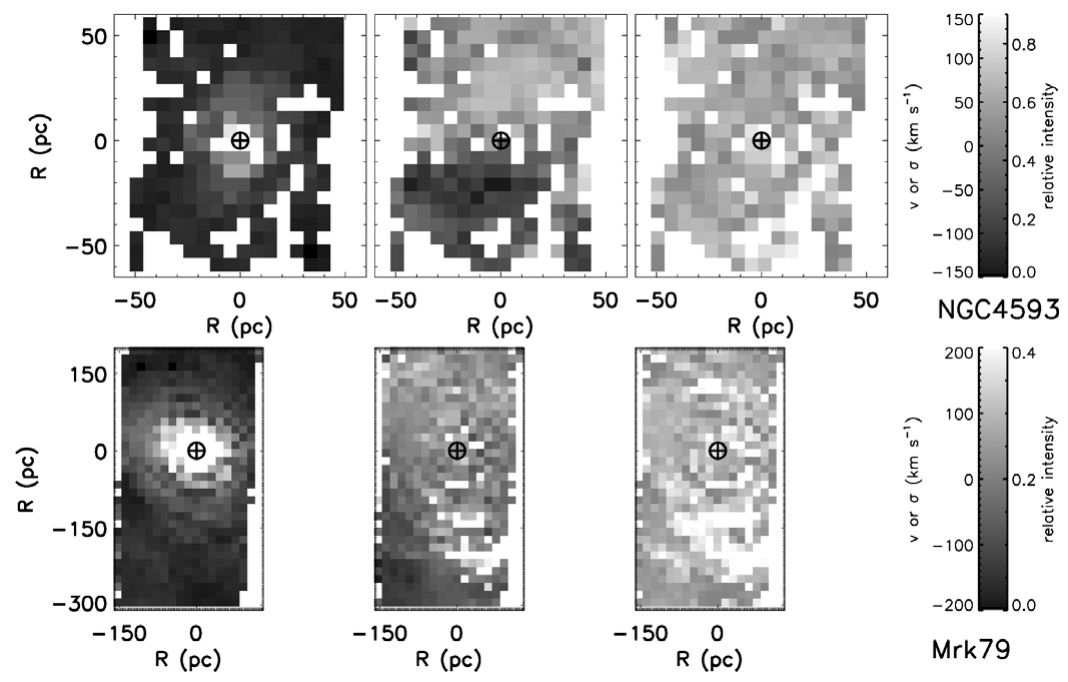

Figure 1. 2-D $\mathrm{H}_{2} 2.1218 \mu \mathrm{m}$ maps of two of the nine new galaxies added to the sample. From left to right the maps are of flux, velocity, and velocity dispersion. Similar maps for the original sample of 9 galaxies are shown by Hicks et al. (2009). North is aligned with the $y$-axis and east is to the left, and the circled cross marks the location of the non-stellar continuum.

concluded that with current models, including those considering radiation pressure from AGN and star formation, stellar winds, and supernovae, it is difficult to explain the observed velocity dispersions.

Comparing the spatial distribution and kinematics of the nuclear molecular gas to that of the nuclear stellar population traced by the CO bandheads, we find that they are very similar on scales of tens of parsecs. In a subsample of these galaxies studied by Davies et al. (2007), an additional stellar component was identified on spatial scales similar to the typical HWHM of $\sim 30 \mathrm{pc}$ of the gas disk from an extrapolation of the stellar light profile from larger radii inward. We also find similar evidence for an additional nuclear stellar component in this expanded sample. The similarity of the gas and stellar kinematics on these scales, including an elevated velocity dispersion in the nuclear stars, leads us to conclude that they are spatially mixed within a vertically thick nuclear disk.

Assuming a gas mass fraction of $10 \%$ (see Hicks et al. 2009 for justification of this assumption), and taking the dynamical mass implied by the gas kinematics (which, in general, agrees with that from the stellar kinematics), we find an average gas mass within a radius of $30 \mathrm{pc}$ of $\sim 10^{7} M_{\odot}$. This implies an average column density in these galaxies of $N_{\mathrm{H}} \sim 5 \times 10^{23} \mathrm{~cm}^{-2}$. However, considering the extinction toward the nuclear stars, assuming both screen and mixed extinction models, implies significantly lower densities, up to an order of magnitude less. This therefore suggests that on these scales the gas distribution is dominated by dense clumps, and that in over half of the galaxies the resulting lower column densities are still great enough to obscure the central AGN.

Based on the spatial distribution and kinematics, as well as the high column densities derived, we conclude that the molecular gas measured on scales of tens of parsecs in the sample of Seyfert galaxies is likely associated with the obscuring "torus" invoked in AGN unification models. Although obscuration of the central AGN is likely to occur on even smaller parsec scales, it also appears to be possible in many cases to obscure the AGN on scales of tens of parsecs. Furthermore, given that the molecular gas and nuclear stellar population are spatially mixed, and the probable dependence of these two components on each other (e.g., Davies et al., these proceedings), we posit that the AGN torus is 

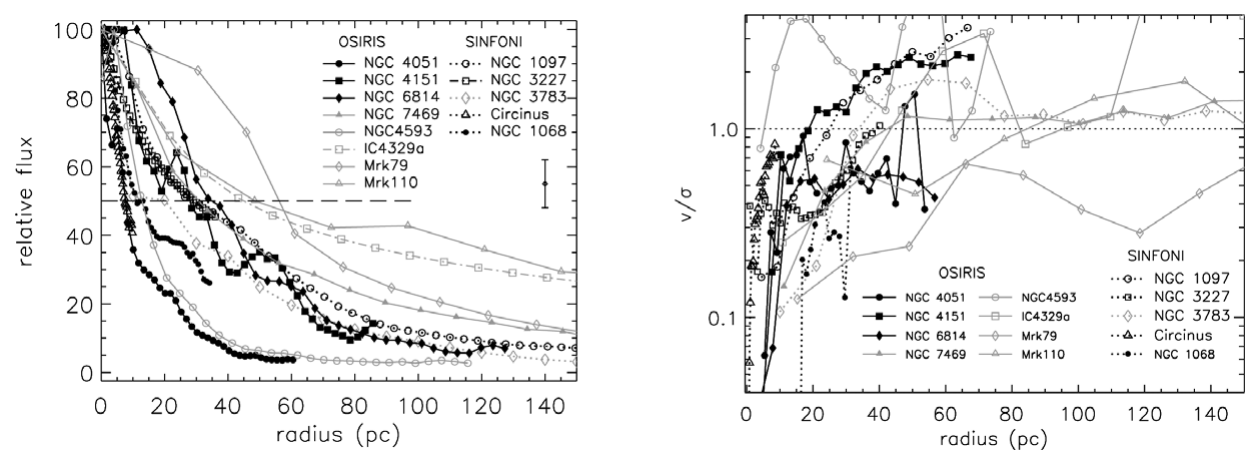

Figure 2. Azimuthally averaged $\mathrm{H}_{2} 2.1218 \mu \mathrm{m}$ properties based on the full 2-D data. Galaxies plotted in black have a well resolved flux distribution, while galaxies plotted in gray are only marginally, or not, resolved. Left: The $\mathrm{H}_{2}$ flux distributions. The horizontal dashed line indicates the HWHM, which for all cases where nuclear emission is resolved is less than 35 pc. For NGC 3227 and IC4329a, the best-fit Sersic function is shown due to the significant asymmetry of their $\mathrm{H}_{2}$ flux distributions. Typically, the standard deviation of the azimuthally averaged flux is $5 \%$, and an error bar representing the typical error of the flux measurements is shown on the right. Right: The $v_{\text {rot }} / \sigma$ ratio as a function of radius for each galaxy as indicated in the legend.

composed of several components over a range of scales (sub-parsec out to at least $\sim 100$ parsecs in Seyfert galaxies) and that these structures are most likely dynamic in nature. In this scenario each component serves different functions and it is the combination of these that satisfies the requirements of the obscuring torus characterized by such AGN unification models.

\section{Estimating $M_{\mathrm{BH}}$ via Modeling of Nuclear Gas Disks}

The properties of the molecular hydrogen gas disks observed in this sample of galaxies suggest that the gas kinematics is dominated by the gravitational potential of the nuclear region and is thus suitable for dynamical modeling. Although there are cases where nongravitational forces impact the gas kinematics (e.g., minor axis outflow in NGC 3227, interaction with the parsec-scale radio jet in NGC 4151), these superimposed components are secondary to the general rotation observed in the gas and these components can be separated. We thus conclude that the molecular gas in the nuclear region of Seyfert galaxies is, in general, a reliable tracer of the gravitational potential, and that, with the spatial resolutions achieved, reliable black hole mass estimates can be obtained from modeling of the resolved gas kinematics.

We are therefore in the process of developing appropriate models to constrain $M_{\mathrm{BH}}$ in the 13 galaxies for which $\mathrm{H}_{2}$ is measured at a high enough signal-to-noise to yield a meaningful estimate (i.e., $\gtrsim 40$ per pixel). The modeling of the gas disk kinematics will follow a procedure similar to that applied by Hicks \& Malkan (2008), which accounts for instrumental effects such as PSF and the emission-line surface brightness. We have also included in the modeling the significant velocity dispersion through the Jeans equation (see Neumayer et al. 2007 for an example of the success of this approach).

As an initial attempt to constrain the black hole mass estimates, we have derived an upper limit from the dynamical mass within as small a radius as possible to reliably measure in the gas kinematics (typically $\sim 35 \mathrm{pc}$ ). Neglecting the velocity dispersion, using $M_{\mathrm{dyn}}=v_{\mathrm{rot}}^{2} R / G$ (where $v_{\text {rot }}$ is the inclination-corrected rotational velocity, $R$ is the radius at which the kinematics is measured, and $G$ is the gravitational constant), we find that for 7 of the 13 galaxies, the reverberation-mapped black hole mass estimates are 

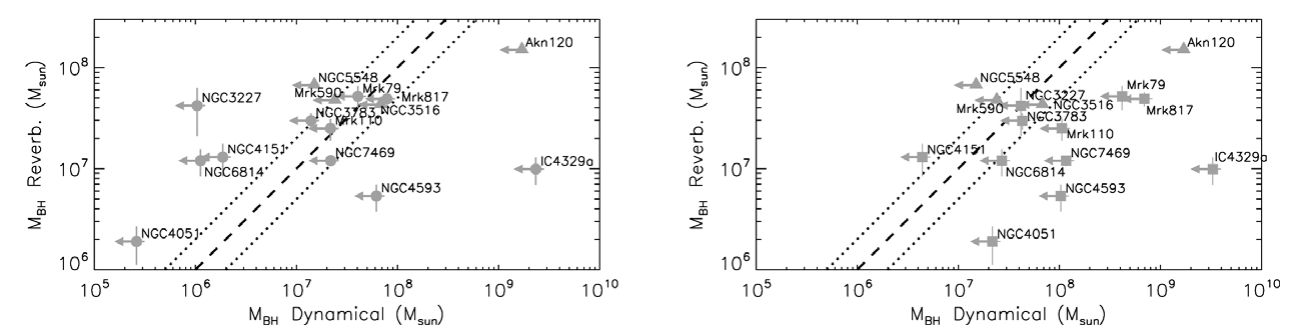

Figure 3. $M_{\mathrm{BH}}$ upper limits based on the dynamical mass within the smallest reliably measured radius (see Table 1) compared to reverberation mapping estimates (equality line is indicated by the dashed curve and the expected error of the latter is shown by the dotted curves). Left: Limits calculated based only on the velocity gradient (triangles are based on stellar kinematics). Right: Gas dynamical masses derived accounting for the velocity dispersion as discussed in the text.

overestimated by as much as an order of magnitude (Figure 3). However, by taking into account the velocity dispersion, we find no indication that reverberation mapping yields estimates with a systematic offset (Figure 3). To account for the velocity dispersion we assume the dispersion arises from macroscopic motions and that we are observing only one dimension of an isotropic distribution, in which case $M_{\mathrm{dyn}}=\left(v_{\mathrm{rot}}^{2}+3 \sigma^{2}\right) R / G$.

\section{Conclusions}

The gas dynamical modeling described above will provide $M_{\mathrm{BH}}$ estimates with an uncertainty expected to be less than $30 \%$. With these $M_{\mathrm{BH}}$ estimates it will then be possible to calibrate the method of reverberation mapping. Furthermore, these $M_{\mathrm{BH}}$ estimates will be compared with estimates obtained via modeling of the stellar kinematics. In addition, up to five more $M_{\mathrm{BH}}$ estimates will also be obtained from modeling of the stellar kinematics in those galaxies where modeling of the gas kinematics is not possible due to weak $\mathrm{H}_{2}$ emission, bringing the full sample to 18 galaxies. Once a reliable calibration of the reverberation mapping method is complete, we will then be able to apply this method, and the secondary methods calibrated against it, with confidence at higher redshifts and in larger samples of galaxies to more fully explore the link between $\mathrm{BH}$ and host galaxy formation and evolution, and the role of the AGN phenomenon therein.

\section{References}

Davies, R. I., Sánchez, F. M., Genzel, R., Tacconi, L. J., Hicks, E. K. S., Friedrich, S., \& Sternberg, A. 2007, ApJ, 671, 138

Ferrarese, L. \& Merritt, D. 2000, ApJ, 539, L9

Gebhardt, K., et al. 2000, ApJ, 539, L13

Gultekin, K., et al. 2009, ApJ, 698, 198

Hicks, E. K. S., Davies, R. I., Malkan, M. A., Genzel, R., Tacconi, L. J., Mueller Sanchez, F., \& Sternberg, A. 2009, ApJ, 696, 448

Hicks, E. K. S. \& Malkan, M. A. 2008, ApJS, 174, 31

Kormendy, J. \& Richstone, D. 1995, ARAA, 33, 581

McGill, K. L., Woo, J., Tommaso, T., \& Malkan, M. A. 2008, ApJ, 673, 703

Neumayer, N. Cappellari, M., Reunanen, J., Rix, H.-W., van der Werf, P., \& Davies, R. I. 2007, ApJ, 671, 1329

Peterson, B. M., et al. 2004, ApJ, 613, 682

Vestergaard, M. 2002, ApJ, 571, 733 